\title{
Vedolizumab treatment in a patient with X-linked agammaglobulinemia, is it safe and efficient?
}

\author{
Şükrü Çekiçç, Taner Özgür², Yasin Karalı1 ${ }^{1}$, Tanju Özkan², Sara Şebnem Kılıç̣̂ \\ Divisions of ${ }^{1}$ Pediatric Immunology and ${ }^{2}$ Pediatric Gastroenterology, Department of Pediatrics, Uludağ University Faculty \\ of Medicine, Bursa, Turkey.E-mail: sebnemkl@uludag.edu.tr \\ Received: 21st January 2019, Revised: 2nd April 2019, Accepted: 10th June 2019
}

SUMMARY: Çekiç Ş, Özgür T, Karalı Y, Özkan T, Kılıç SŞ. Vedolizumab treatment in a patient with X-linked agammaglobulinemia, is it safe and efficient? Turk J Pediatr 2019; 61: 937-940.

The loss of inflammatory regulation resulting from the absence of B-lymphocytes leads to a risk for autoimmune and autoinflammatory complications. There is no data on the use of Vedolizumab in patients with $\mathrm{X}$-linked agammaglobulinemia (XLA) as well as children with another primary immunodeficiency (PID) diseases. A 4-year-old boy was admitted to our clinic with a history of recurrent respiratory tract infections. He was diagnosed with XLA based on extremely low immunoglobulins, very low level of B cells, genetic mutation of BTK gene, and family history. At the age of 8 , he suffered from intermittent fever attacks, abdominal pain, weakness, oral aft, and weight loss. His clinical and laboratory features were consistent with inflammatory bowel disease. Histopathological examination of the biopsy material obtained from terminal ileum, colon and cecum showed Crohn's disease. Initially, he was treated with prednisolone and infliximab. Because of the lack of response, infliximab treatment was switched to adalimumab. Terminal ileum was resected to relieve obstruction complication. Although he had been treated with adalimumab, a significant improvement was not observed. Vedolizumab (Entyvio ${ }^{\mathrm{TM}}$ ), a humanized monoclonal antibody $\alpha 4 \beta 7$ integrin-receptor antagonist, was commenced. After treatment with vedolizumab, his fever and abdominal pain attacks reduced, his total daily calorie intake increased and weight gain improved. He began to walk again and continued his school education properly. No side effects were observed in 18 months. This is the first immunocompromised child treated with vedolizumab. The symptoms of the patient receded and no side effect were seen during the treatment.

Keywords: vedolizumab, $X$ linked agammaglobulinemia, crohn's disease, adalimumab.

X-linked agammaglobulinemia (XLA) or Bruton disease caused by mutations in the gene coding for Bruton tyrosine kinase (BTK) is a rare immunodeficiency characterized by severe antibody deficiency and recurrent infections. ${ }^{1} \quad$ Autoinflammatory diseases are part of characteristic findings in many primary immunodeficiencies (PIDs). ${ }^{2}$ The incidence of inflammatory disease in XLA is higher than in normal population. ${ }^{2}$ The loss of inflammatory regulation resulting from the absence of B-lymphocytes leads a risk for autoimmune and autoinflammatory complications. ${ }^{1}$ Gastrointestinal system (GIS) related complaints have been reported in $35 \%$ of these patients. ${ }^{3}$ The frequencies of inflammatory bowel disease (IBD) in different XLA cohorts were reported as $3.6-3.8 \% .^{4}$

Vedolizumab is an anti-integrin antibody that is indicated in IBD resistant to anti-TNF drugs. There is no data on the use of vedolizumab in 
patients with XLA as well as children with other PID diseases.

\section{Case Report}

A 4-year-old boy was admitted to our clinic with a history of recurrent respiratory tract infections. All immunoglobulins (Ig) were extremely low (IgG $<154 \mathrm{mg} / \mathrm{dl}$, IgA $<25.4 \mathrm{mg} /$ $\mathrm{dl}, \operatorname{IgM}<17.8 \mathrm{mg} / \mathrm{dl}$ ) with the absence of $\mathrm{B}$ cells (CD19 and CD20 was 0.3\%). His cousin (the child of his mother's sister) also had XLA. A missense point mutation $\mathrm{c} 562 \mathrm{C}>\mathrm{T}$ in exon 17 of BTK gene was identified in both affected cousins. The patient was commenced on regular intravenous immunoglobulin (IVIG) treatment every 3 weeks. At the age of 8 , he suffered from intermittent fever attacks, abdominal pain, weakness, oral aft, and weight loss. His laboratory investigation showed anemia and thrombocytosis (766.000/ $\left.\mathrm{mm}^{3}\right)$. While erythrocyte sedimentation rate (ESR) $(29 \mathrm{~mm} / \mathrm{h}$, normal range: $2-20 \mathrm{~mm} / \mathrm{h})$, C- reactive protein (CRP) $(9.9 \mathrm{mg} / \mathrm{dl}$, normal range $<0,5 \mathrm{mg} / \mathrm{dl})$, fibrinogen $(526 \mathrm{mg} / \mathrm{dl}$, normal range: $180-400 \mathrm{mg} / \mathrm{dl}$ ), serum amyloid A $(318 \mathrm{mg} / \mathrm{L}$, normal range $<6.8 \mathrm{mg} / \mathrm{L})$ levels were found high, albumin $(2.7 \mathrm{gr} / \mathrm{dl}$, normal, range: $4-5 \mathrm{gr} / \mathrm{dl})$ and prealbumin $(0.07 \mathrm{gr} / \mathrm{L}$, normal range: $0.18-0.45$ ) levels were found low. Biochemical analyses of the stool were normal except for the impaired carbohydrate digestion. Stool culture, antigen detection assays and microscopy excluded any pathogenic bacteria, virus, parasites, Giardia and Cryptosporidium. Cytomegalovirus PCR was negative in both blood and intestinal biopsy specimens. Magnetic resonance (MR) enterography showed enlarged mesenteric lymph nodes, thickened walls of jejunum, narrowed lumen and prestenotic dilatation of the jejunum (Fig. 1). The histologic examination of endoscopic biopsies obtained from terminal ileum, colon and cecum mucosa revealed nonspecific chronic active inflammatory infiltration.

Initially, the patient was treated with steroids and azathioprine with partial response. Infliximab was started $5 \mathrm{mg} / \mathrm{kg}$ at weeks 0,2 , and 6 induction regimen and followed by maintenance dosing every 8 weeks. At the beginning of infliximab treatment, his weight increased, and fever attacks receded. At the 8th month of infliximab treatment, his complaints re-appeared, and infliximab was switched to adalimumab. Total parenteral nutrition (TPN) was started intravenously because of limited oral feeding. His complaints increased markedly, and he lost the ability to walk due to severe weight and muscle mass loss. Proximal jejunal resection was performed to relieve the obstruction in the jejunum. Histopathological examination of the specimen was consistent with Crohn's disease (CD). Besides adalimumab (40 mg/every 2 weeks), the patient was treated by mesalamine, oral budesonide and azathioprine in the certain period since the onset of disease.

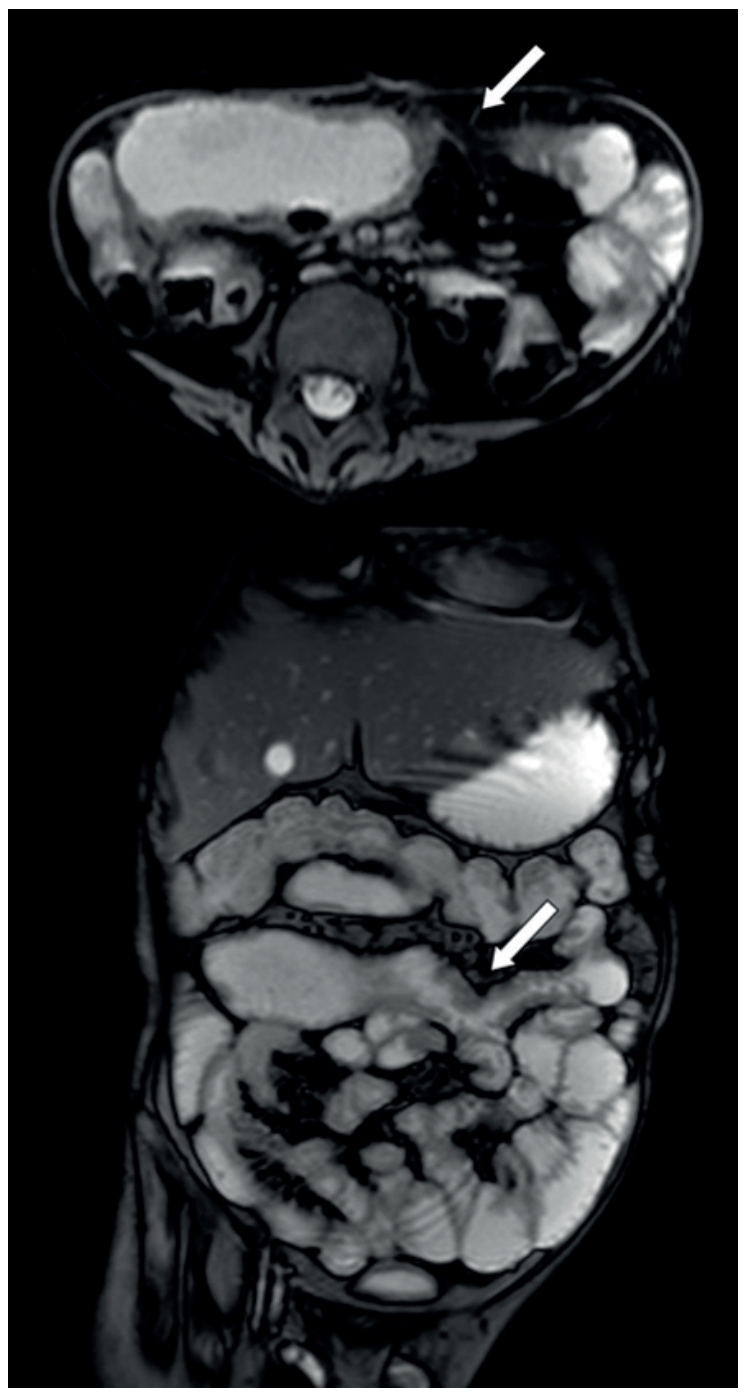

Fig. 1. The appearance of stricture and prestenotic dilatation in transverse and horizontal sections of jejunum in magnetic resonance enteroscopy. 
Finally, vedolizumab was initiated with a 30 -minute or longer $300 \mathrm{mg}$ infusion at weeks 0,2 , and 6 and continued every 8 weeks after that. After the vedolizumab, his fever and abdominal pain attacks reduced after the first month of this therapy, and his total daily calorie intake increased and weight gain improved (Fig. 2). The Pediatric Crohn's Disease Activity Index ${ }^{5}$ was 77.5 ( $\geq 40$ Severe $\mathrm{CD})$ before vedolizumab therapy, followed by 22.5 and 20 (10-27.5 mild CD) at the 6th and 12th months of the therapy, respectively. The hospitalization number was 11 annually before vedolizumab treatment depending on CD. However, during the 18 months of treatment, he continued his school education regularly and the patient did not need to be hospitalized.

He began to walk again and continued his school education properly. No side effects were observed in 18 months. Re-evaluation by endoscopy was not performed because of the difficulty to reach a patchy lesion in the small intestine and to avoid invasive procedures as much as possible.

The informed consent was received from the parents for publication.

\section{Discussion}

Although the pathogenesis of IBD may be different in immunocompromised patients, it is suggested that IBD in immunocompromised patients should be treated like immunocompetent patients. ${ }^{6}$ The treatment of GIS symptoms in XLA patients is limited with IVIG, corticosteroids, and TPN in The database of United States Immunodeficiency Network. ${ }^{3}$

Small intestinal strictures and transmural intestinal fissures in the histological examination can be seen in CD with XLA, similar to immunocompetent patients with $\mathrm{CD}^{7}$ However, contrary to $\mathrm{CD}$ in immunocompetent patients, granulomas or plasma cells may not be seen in patients with XLA, because there are no germinal centers in the intestinal-associated lymphoid tissues, and nodular lymphoid hyperplasia does not develop in XLA patients. ${ }^{6}$

The use of infliximab has been reported in cases of other immunodeficiencies to treat granulomatous diseases in both extraintestinal and intestinal locations. Davey et al. ${ }^{8}$ reported an XLA patient with granulomatous small bowel enteropathy that was treated successfully with infliximab without serious side-effects. Although our patient responded well to infliximab treatment at the beginning of the disease, response to infliximab decreased in the follow-up period. No side effect associated with infliximab was seen.

There are few studies showing the treatment efficacy of adalimumab on patients with CD associated with PIDs. ${ }^{9}$ The data related to use of Adalimumab in XLA is very limited. Adalimumab was used for one year without any side effects in our patient. However, its benefit was limited.

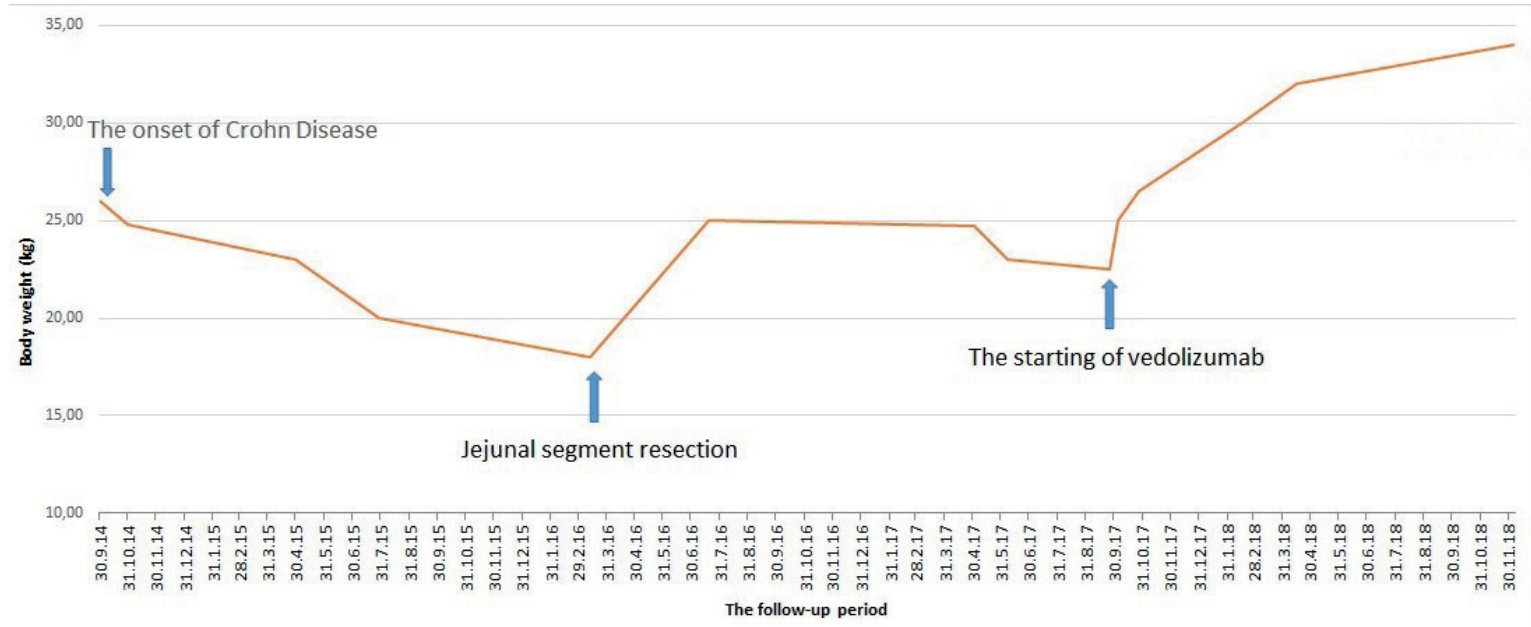

Fig. 2. The overview of body weight of the patient. 
Vedolizumab $\left(\right.$ Entyvio $^{\mathrm{TM}}$ ), is a humanized monoclonal antibody $\alpha 4 \beta 7$ integrin-receptor antagonist, it blocks the interaction between a4ß7 integrin found on T-cells surface and MAdCAM-1 which is expressed on the endothelial surface of venules within the gastrointestinal tract. Therefore, it selectively impacts on gut-specific lymphocyte trafficking. ${ }^{10}$ Boland et al. ${ }^{11}$ have recently reported three adult patients with CVID having IBD treated with vedolizumab successfully. While vedolizumab has been reported as an effective therapy alternative in adult patients with IBD, there is limited data regarding the dosage and treatment duration on pediatric cases in the literature. To our knowledge, this is the first pediatric patient with PID treated with vedolizumab. The symptoms of our patient receded shortly after using vedolizumab treatment and no side effects were observed during 18 months of the treatment.

CD can be severe and atypical in XLA. Infliximab and adalimumab can be well tolerated in children with XLA. Vedolizumab appears to be an effective and safety option in treatment-resistant children with CD and PID.

\section{REFERENCES}

1. Aneja A, Chhabra A. Bruton Agammaglobulinemia. [Updated 2017 Oct 9]. In: StatPearls [Internet]. Treasure Island (FL): StatPearls Publishing; 2018 Jan. Available from: http:// europepmc.org/books/NBK448170;jsessionid= CC9B9257254CF5E80DF7FF32D091BA

2. Hernandez-Trujillo VP, Scalchunes C, CunninghamRundles C, et al. Autoimmunity and inflammation in X-linked agammaglobulinemia. J Clin Immunol 2014; 34: 627-632.
3. Barmettler S, Otani IM, Minhas J, et al. Gastrointestinal manifestations in X-linked agammaglobulinemia. J Clin Immunol 2017; 37: 287-294.

4. Walter JE, Farmer JR, Foldvari Z, Torgerson TR, Cooper MA. Mechanism-based strategies for the management of autoimmunity and immune dysregulation in primary immunodeficiencies. J Allergy Clin Immunol Pract 2016; 4: 1089-1100.

5. Hyams J, Markowitz J, Otley A, et al; Pediatric Inflammatory Bowel Disease Collaborative Research Group. Evaluation of the pediatric crohn disease activity index: a prospective multicenter experience. J Pediatr Gastroenterol Nutr 2005; 41: 416-421.

6. Agarwal S, Mayer L. Pathogenesis and treatment of gastrointestinal disease in antibody deficiency syndromes. J Allergy Clin Immunol 2009; 124: 658664.

7. Washington K, Stenzel TT, Buckley RH, Gottfried MR. Gastrointestinal pathology in patients with common variable immunodeficiency and X-linked agammaglobulinemia. Am J Surg Pathol 1996; 20: 1240-1252.

8. Davey PT, Tan CJ, Gardiner K. The use of infliximab in X-linked agammaglobulinaemia associated enteropathy. Ann R Coll Surg Engl 2014; 96: e5-e6.

9. Robles-Marhuenda A, Cobo-Ibáñez T, Ríos-Blanco J, Arnalich-Fernández F. AB0927 biological drugs use in primary immunodeficiencies. Ann Rheum Dis 2016; 75(Suppl 2): 1218.

10. Fedyk ER, Wyant T, Yang LL, et al. Exclusive antagonism of the $\alpha 4 \beta 7$ integrin by vedolizumab confirms the gut-selectivity of this pathway in primates. Inflamm Bowel Dis 2012; 18: 2107-2119.

11. Boland BS, Riedl MA, Valasek MA, Crowe SE, Sandborn WJ. Vedolizumab in patients with common variable immune deficiency (CVID) and gut inflammation. Am J Gastroenterol 2017; 112: 1621. 